\title{
Of Fish and Men
}

Drug Screening in Scn 1a Zebrafish Mutant Identifies Clemizole as a Potential Dravet Syndrome Treatment. Baraban SC, Dinday MT, Hortopan GA. Nat Commun 2013;4:2410. doi:10.1038/ncomms3410.

Dravet syndrome is a catastrophic pediatric epilepsy with severe intellectual disability, impaired social development and persistent drug-resistant seizures. One of its primary monogenic causes are mutations in $\mathrm{Na}_{\mathrm{v}} 1.1$ (SCN1A), a voltage-gated sodium channel. Here we characterize zebrafish $\mathrm{Na}_{\mathrm{v}} 1.1$ ( $\left.s \mathrm{cn} 1 \mathrm{Lab}\right)$ mutants originally identified in a chemical mutagenesis screen. Mutants exhibit spontaneous abnormal electrographic activity, hyperactivity and convulsive behaviours. Although scn $1 \mathrm{Lab}$ expression is reduced, microarray analysis is remarkable for the small fraction of differentially expressed genes ( $3 \%)$ and lack of compensatory expression changes in other scn subunits. Ketogenic diet, diazepam, valproate, potassium bromide and stiripentol attenuate mutant seizure activity; seven other antiepileptic drugs have no effect. A phenotype-based screen of 320 compounds identifies a US Food and Drug Administration-approved compound (clemizole) that inhibits convulsive behaviours and electrographic seizures. This approach represents a new direction in modelling pediatric epilepsy and could be used to identify novel therapeutics for any monogenic epilepsy disorder.

\section{Commentary}

Dravet syndrome (DS) is a severe pediatric epilepsy that presents with multiple seizure types commonly resistant to pharmacologic treatment, as well as intellectual disability, behavioral abnormalities, gait and motor dysfunction, and increased mortality (1). In most cases, the disease is caused by heterozygous de novo mutations or gene deletions of SCN1A, the gene encoding the pore-forming protein $\mathrm{Na}_{\mathrm{v}} 1.1$ of the voltage-gated sodium channel (VGSC) (2).

DS is considered an epileptic encephalopathy, meaning that seizures contribute to the progressive worsening of the neurologic condition (1). Unfortunately, most available anticonvulsants are ineffective in controlling the seizures; some anticonvulsants, such as phenytoin, carbamazepine, and lamotrigine, are sodium channel blockers and may worsen seizure control. Among the medications that have shown some efficacy are valproate, clobazam, clonazepam, topiramate, levetiracetam, bromides, and stiripentol (3). The ketogenic diet is also useful and may reduce seizure frequency in up to $75 \%$ of patients (4). These treatments are neither fully effective nor curative, however, and thus a need exists for finding better therapies for DS. The search for such new compounds would be greatly aided by the availability of a DS model amenable to high-throughput screening (HTPS).

Available models of DS include Scn1 a knock-out mice (5), mice engineered with a human DS SCN1A mutation (6), and DS patient-induced pluripotent-stem cell (iPSC)-derived neurons $(7,8)$. While the murine models reproduce many of the behav-

Epilepsy Currents, Vol. 14, No. 2 (March/April) 2014 pp. 97-98

(c) American Epilepsy Society

OPEN 2 ACCESS Freely available online ioral and electrographic characteristics of the disease, breeding is often difficult and takes substantial time, seizure phenotypes vary considerably between different mouse genetic backgrounds, and screening techniques are not easily scalable. The DS iPSC model has the advantage of being patient derived and shows in vitro electrophysiological abnormalities, but at present no consensus exists on the best method to monitor responses to pharmacologic treatments in this model.

The study by Baraban et al. aims to investigate the feasibility of using zebrafish as an animal model of DS to investigate disease mechanisms and the feasibility of HTPS of pharmacologic compounds. Given the short breeding times and the possibility of simultaneously monitoring dozens of fish, this is an intriguing idea. The authors take advantage of a previously described mutant fish line carrying a homozygous mutation in the gene Scn 1Lab (9), which shares $77 \%$ identity with the human SCN1A gene. Because the zebrafish genome underwent a duplication during evolution, the fish have two genes homologous to SCN1A: SCn 1Lab and SCn 1Laa. Thus, a homozygous mutation in one of these genes would be equivalent to a heterozygous condition in the human.

Through the use of quantitative PCR and in situ hybridization, the authors demonstrate that $S C n 1 L a b$ is expressed during early CNS development. The mutation causes a reduction in the expression levels of this gene, while those of other VGSC genes are not affected. Fortuitously and for unknown reasons, the mutant fish exhibit hyperpigmentation, facilitating the recognition of mutants from their wild-type siblings. Microarray and quantitative reverse transcriptase-polymerase chain reaction (RT-PCR) demonstrated mild changes in the expression level of 1,099 genes, including some associated with CNS disorders, and this database should provide a valuable resource for future studies. 


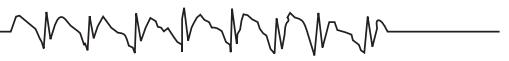

Behaviorally, the mutant zebrafish exhibit premature spontaneous death between 10 and 12 days post fertilization (mid larval/prejuvenile stage). They also have elevated levels of swim activity, spontaneous convulsions, and rapid undirected movements, while none of these behaviors are observed in wild-type siblings. Such abnormal movements in larvae exposed to the proconvulsant drug pentylenetetrazole have been reported by the authors in a previous study, suggesting that these movements correspond to seizures (10). Of importance, zebrafish movement can be measured with the use of automated tracking devices. For recording forebrain extracellular field potentials, the larvae were immobilized in agar (to obviate the need for anesthetics); then a glass microelectrode was inserted in the forebrain. Both interictal bursts and largeamplitude ictal events were recorded from mutants but were never observed in controls.

After confirming the presence of behavioral and electrographic abnormalities consistent with seizures, the authors performed a pharmacologic validation by measuring the effect of currently available anticonvulsant medications on the electrographic activity and swimming behavior of mutant larvae. Similar to the lack of efficacy in humans, many drugs had no effect on burst frequency, burst duration, cumulative seizure duration, and seizure-like behavior; however, valproate, diazepam, potassium bromide, and stiripentol caused significant decreases in burst frequency, time spent in seizures, and seizure behavior. To examine the effect of ketosis as a proxy for the ketogenic diet, the authors added ketogenic substrates to the water and checked for abnormal movements with locomotion tracking. While control larvae had no change in behavior when exposed to the ketogenic compounds, mutant larvae exhibited a reduction in seizure-like behavior. Collectively, these results suggest that $S c n 1 L a b$ mutant zebrafish are able to recapitulate some of the behavioral, electrographic, and pharmacologic characteristics of DS.

Having validated their animal model, the authors then subjected the mutant fish to an HTPS of a repurposing drug library to identify new compounds that ameliorate the seizure-like behavior in the locomotion tracking assay. The authors placed fish in 96-well plates and automatically tracked swim activity at baseline and then after exposure to 320 test compounds previously approved by the FDA and toxicology tested. After several rounds of testing at various concentrations, they identified four compounds that reduced spontaneous seizures and swimming speed to normal levels without showing toxicity. Those four compounds were then tested for their effect on electrographic activity. Three of the compounds proved to be muscle relaxants that abolished abnormal seizure-like behavior but not electrographic ictal activity. Only clemizole, an antihistamine and NS4B RNA-binding inhibitor, was effective in normalizing both swimming behavior and electrographic activity. To confirm the anticonvulsive properties of clemizole, the authors then tested its effect on the pentylenetetrazole zebrafish model, where it again proved capable of reducing seizures.

This study not only validates a new vertebrate animal model of DS but also supports the use of this model for HTPS of new pharmacologic treatments. The model and screening methods used led to the reliable detection of both anticonvul- sant efficacy of the compounds and toxicity at the organismal level. One of the most exciting findings of the article is the suggestion that a currently FDA-approved compound might be a feasible treatment for this devastating disease. Whether clemizole will live up to this promise will require testing in mammalian models and subsequent clinical trials. Future studies will also need to define the mechanism of epilepsy in the zebrafish DS model, a very important question since the current mouse and iPSC models may differ in hyperexcitability mechanisms (5-8). It will also be interesting to explore whether Scn $1 \mathrm{Laa}$ mutants also serve as DS models and, if so, whether differences exist with the current model. Nonetheless, the present works suggests that zebrafish models of genetic epilepsies are very promising for HTPS to identify novel epilepsy therapies. One can envision future preclinical strategies of antiepileptic drug development that combine screening and testing in zebrafish, rodent, and patient-derived neuronal models of epilepsy. The hope is that this approach will translate to improved success in clinical trials of promising drug candidates.

by Gustavo A. Patino, MD PhD, Jack M. Parent, MD

\section{References}

1. Akiyama M, Kobayashi K, Ohtsuka Y. Dravet syndrome: A genetic epileptic disorder. Acta Med Okayama 2013;66:369-376.

2. Wang JW, Shi XY, Kurahashi H, Hwang SK, Ishii A, Higurashi N, Kaneko S, Hirose S. Prevalence of SCN1A mutations in children with suspected Dravet syndrome and intractable childhood epilepsy. Epilepsy Res 2012;102:195-200.

3. Chiron C, Dulac O. The pharmacologic treatment of Dravet syndrome. Epilepsia 2011;52(suppl 2):72-75.

4. Nabbout R, Copioli C, Chipaux M, Chemaly N, Desguerre I, Dulac O, Chiron C. Ketogenic diet also benefits Dravet syndrome patients receiving stiripentol: A prospective pilot study. Epilepsia 2011;52:e54-e57. http://onlinelibrary.wiley.com/doi/10.1111/j.15281167.2011.03107.x/full. Accessed November 27th 2013.

5. Yu FH, Mantegazza M, Westenbroek RE, Robbins CA, Kalume F, Burton KA, Spain WJ, McKnight GS, Scheuer T, Catterall WA. Reduced sodium current in GABAergic interneurons in a mouse model of severe myoclonic epilepsy in infancy. Nat Neurosci 2006;9:1142-1149.

6. Ogiwara I, Miyamoto H, Morita N, Atapour N, Mazaki E, Inoue I, Takeuchi T, Itohara S, Yanagawa Y, Obata K, Furuichi T, Hensch TK, Yamakawa K. Na $\mathrm{N}_{\mathrm{v}} 1.1$ localizes to axons of parvalbumin-positive inhibitory interneurons: A circuit basis for epileptic seizures in mice carrying an Scnl la gene mutation. J Neurosci 2007;27:5903-5914.

7. Jiao J, Yang Y, Shi Y, Chen J, Gao R, Fan Y, Yao H, Liao W, Sun XF, Gao S. Modeling Dravet syndrome using induced pluripotent stem cells (iPSCs) and directly converted neurons. Hum Mol Genet 2013;22:42414252.

8. Liu Y, Lopez-Santiago LF, Yuan Y, Jones JM, Zhang H, O'Malley HA, Patino GA, O'Brien JE, Rusconi R, Gupta A, et al. Dravet syndrome patient-derived neurons suggest a novel epilepsy mechanism. Ann Neurol 2013;74:128-139.

9. Schoonheim PJ, Arrenberg AB, Del Bene F, Baier H. Optogenetic localization and genetic perturbation of saccade-generating neurons in zebrafish. J Neurosci 2010;30:7111-7120.

10. Baraban SC, Dinday MT, Castro PA, Chege S, Guyenet S, Taylor MR. A large-scale mutagenesis screen to identify seizure-resistant zebrafish. Epilepsia 2007:48:1151-1157. 